\title{
Unusual delayed presentation of diaphragmatic hernia complicated by transverse colon and total small-bowel obstruction after postoperative chemotherapy of esophageal cancer
}

\author{
This article was published in the following Dove Press journal: \\ Therapeutics and Clinical Risk Management \\ 6 June 2017 \\ Number of times this article has been viewed
}

\section{Yanlai Sun ${ }^{1,2, *}$ \\ Lei Yin ${ }^{2}, *$ \\ Hongfan Xue ${ }^{2}$ \\ Haipeng Wang ${ }^{2}$ \\ Zengjun $\mathrm{Li}^{2}$ \\ Jinming $\mathrm{Yu}^{3}$}

'Post-doctoral Research Station, Tianjin Medical University, Tianjin, ${ }^{2}$ Department of Gastrointestinal Cancer Surgery, ${ }^{3}$ Department of Radiation Oncology, Shandong Cancer Hospital Affiliated to Shandong University, Shandong Academy of Medical Sciences, Jinan, People's Republic of China

*These authors equally contributed to this work
Correspondence: Zengjun Li

Department of Gastrointestinal Cancer Surgery, Shandong Cancer Hospital Affiliated to Shandong University, Shandong Academy of Medical Sciences, 440 Jiyan Road, Jinan 250I I7, People's Republic of China

Tel/fax +8653I 67626075

Email lizengjun676@I63.com

Jinming $Y u$

Department of Radiation Oncology, Shandong Cancer Hospital Affiliated to Shandong University, Shandong Academy of Medical Sciences, 440 Jiyan Road, Jinan 250I 17, People's Republic of China Email sdyujinming@।63.com

\begin{abstract}
Diaphragmatic hernia (DH) is defined as the passage of abdominal contents into the chest cavity through a defect in the diaphragm. DH occurs after chest or abdominal surgery, and is very rare and sporadically reported in the literature. However, the complications are significant and put the patient at great risk. The aim of the present report was to describe a special case with postesophagectomy diaphragmatic hernia (PDH) because of its appearance during chemotherapy and confusion of the symptoms with the side effects of chemotherapy. A high index of suspicion needs to be maintained in clinical practice.
\end{abstract}

Keywords: diaphragmatic hernia, postoperative chemotherapy, esophageal cancer, complications

\section{Introduction}

Diaphragmatic hernia (DH) has been described as a rare but potentially lethal complication after open and minimally invasive thoracoabdominal surgery for esophageal or gastric cancer. ${ }^{1} \mathrm{DH}$ can develop in the early postesophagectomy stage or as a complication during the late stage. The incidence of DH after traditional open esophagectomy has been reported to be between $0.4 \%$ and $6 \% .^{2}$ Diagnosis of $\mathrm{DH}$ is not easy, but it is often associated with life-threatening conditions. ${ }^{3}$ Cisplatin-based chemotherapy regimens are commonly administered to patients with advanced or metastatic esophageal cancer. ${ }^{4}$ It is well known that cancer patients undergoing chemotherapy often experience nausea, vomiting, and loss of appetite. Herein, we report an exceedingly rare case of intrathoracic incarceration of the transverse colon (partial) and small bowel (total) in a patient with DH after postoperative chemotherapy. To our knowledge, postesophagectomy intrathoracic incarceration of transverse colon and small bowel has not been described before. This report aims to raise awareness of this complication in patients undergoing chemotherapy and discuss how to prevent and manage this complication.

\section{Case presentation}

A 48-year-old male underwent an uneventful transthoracic esophagectomy for pT3N1M0 squamous cell carcinoma in the esophagus 3 months prior. The left lateral diaphragmatic incision to enlarge the hiatus in order to mobilize the stomach up into the cervical incision and anastomosed to the proximal esophageal stump was administered. Reconstruction was performed on the aortic arch in the left thoracic 
cavity. The postoperative recovery was smooth without any complications. Two courses of postoperative adjuvant chemotherapy, which consisted of docetaxel (60 mg intravenous [iv] drip day 1 and day 8) and cisplatin (40 mg iv drip days $1-3$ ), were administered due to esophageal cancer. The patient had a mild gastrointestinal reaction. The bone marrow was not significantly suppressed during chemotherapy. He was admitted and referred to the hospital for the third course of postoperative chemotherapy.

In the first 4 days of chemotherapy, the patient was off food, had nausea and vomited. During admission, his routine laboratory values were normal. On abdominal examination, the abdomen was flat and without any signs of peritonitis. Bowel sounds were present, but somewhat increased. Upon review of the previous chemotherapy effects on the patient, we hypothesized that the patient's discomfort was caused by the side effects of chemotherapy. He was treated with anisodamine $(10 \mathrm{mg})$ and metoclopramide $(20 \mathrm{mg})$ by intramuscular injection, and the symptoms improved. The following day, the patient suddenly appeared confused and had dyspnea. The physical examination revealed hyperpnea and cyanosis of the lips. Auscultation revealed crackles in both lung fields but there were no bowel sounds. The electrocardiograph monitoring showed heart rate (HR) 156/bpm, blood pressure (BP) 137/88 mmHg, and $\mathrm{SpO}_{2}$ 55\%. These indicated a need for resuscitation to patient and immediate bedside resuscitation was performed. The patient was given sputum aspiration, endotracheal intubation, and tracheal lavage. In addition, a large amount of gastric content was removed through a nasogastric tube suction at the bedside, and the patient's condition improved and became stable.

To confirm the cause of vomiting, a chest radiograph, barium enema, and chest and abdomen computed tomography (CT) scans were performed, which showed a left-sided DH, with herniation of the small bowel and transverse colon into the left side of the chest and a mediastinal shift (Figures 1 and 2). The patient was taken to the operating room where the transverse colon (partial) with the small bowel (total) had herniated through the enlarged hiatus and migrated inside the left pleural cavity (Figure 3). There were no adhesions between the bowel loops and the surrounding structures. The small bowel and transverse colon were carefully relocated into the abdominal cavity. Partial ischemic and necrotic intestinal segment resection for bowel strangulation, end-to-end intestinal anastomosis, and a proximal end transverse colon neostomy were performed. After hernia reduction, the hiatal defect was repaired directly with no mesh. The postoperative course was uneventful, and the patient's condition improved well. He was discharged 14 days after admission.

\section{Consent}

Written informed consent was obtained from the patient for publication of this case report and any accompanying images.
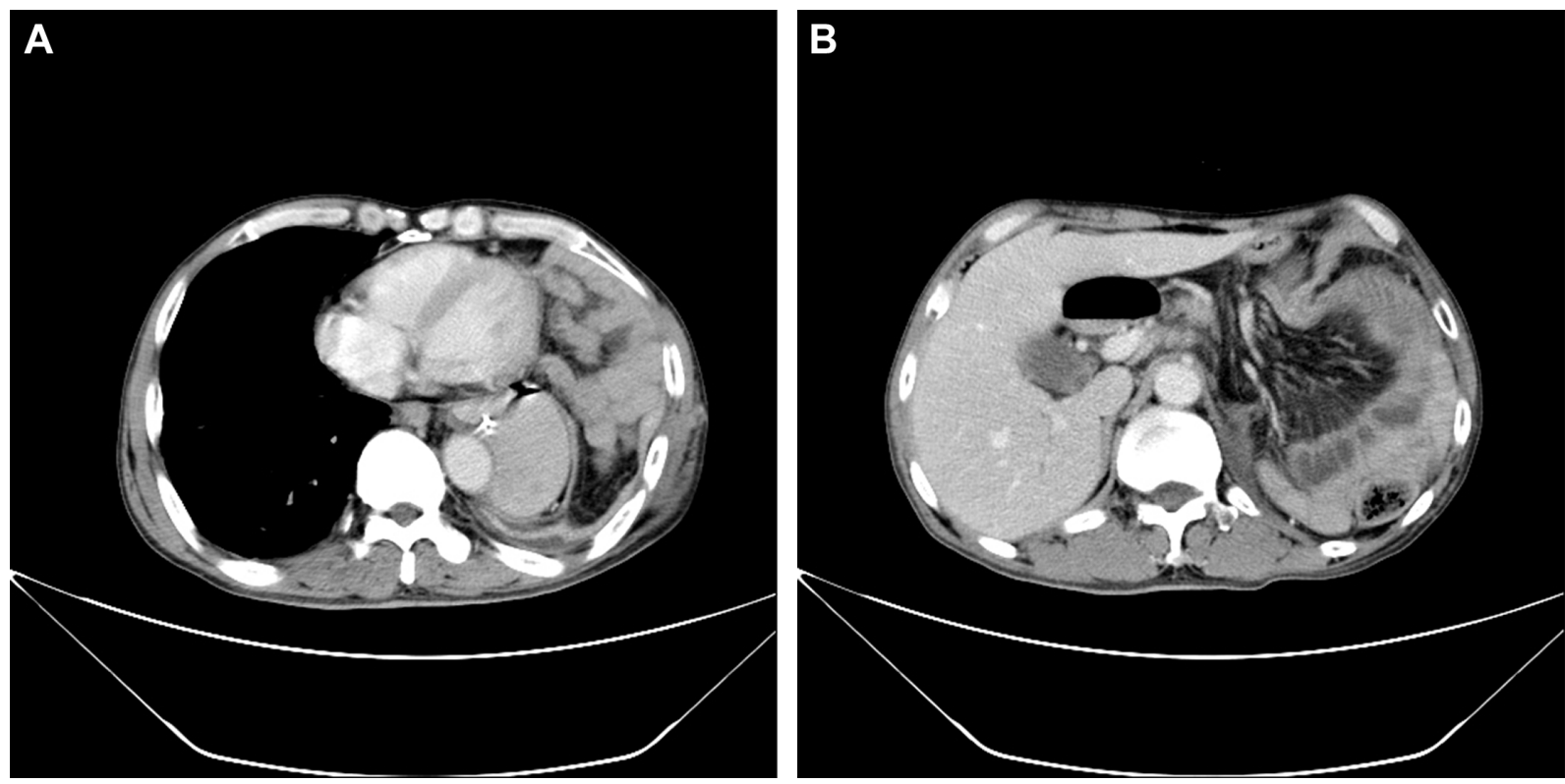

Figure I (A) A transverse computed tomography scan of the patient's chest on admission showing a large left-sided diaphragmatic hernia with bowel loops herniating into the left side of the chest, causing a mediastinal shift and resulting in small-bowel obstruction. (B) Axial CT of the abdomen showed intraperitoneal mesenteric folds. Abbreviation: CT, computed tomography. 


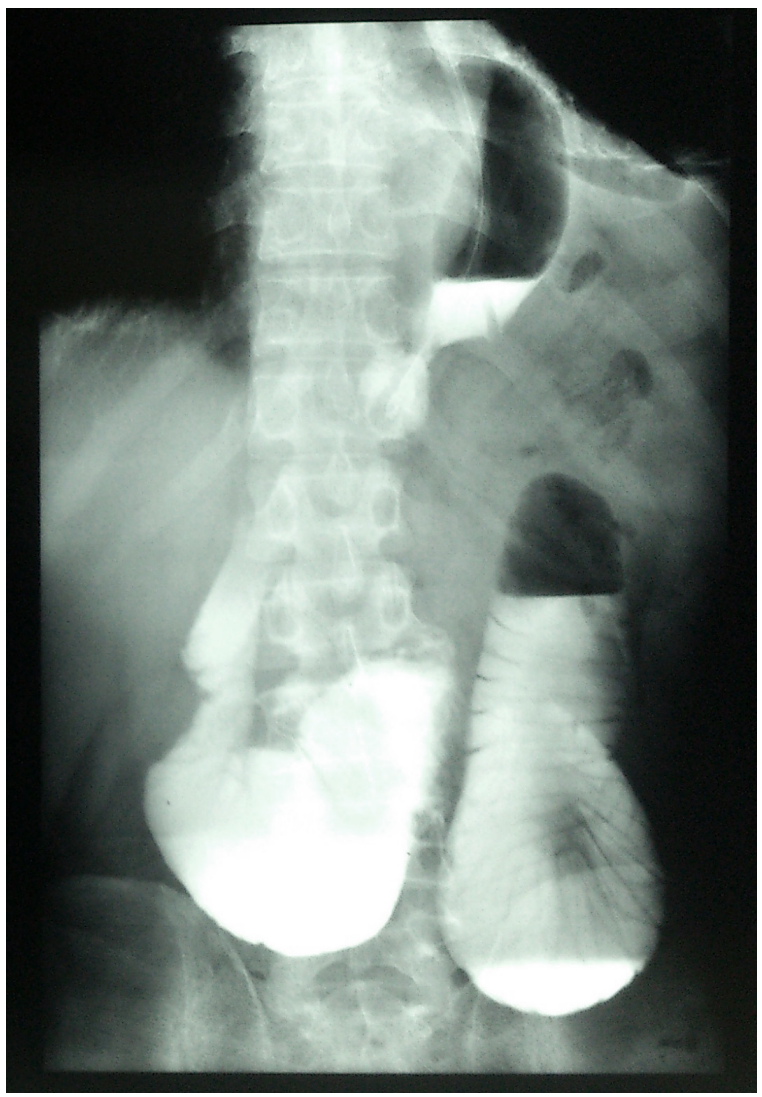

Figure 2 Preoperative barium enema shows dilated intestinal segments detected above the line of the diaphragm. The mediastinum is shifted to the right side.

\section{Discussion}

Esophagectomy is a major procedure with high rates of morbidity, including postesophagectomy diaphragmatic hernia (PDH). PDH presents a major challenge to the surgeon because it may lead to serious potential complications, such as volvulus, strangulation, and perforation. The reports of DHs after esophagectomy for esophageal and gastric cancers are rare. To date, a total of $\sim 30$ cases of early and late DH after esophagectomy for esophageal cancer have been found in English language literature. ${ }^{5}$ The content of PDH most often includes the stomach, followed by the large and small intestines. ${ }^{6}$ However, no case developed a DH during chemotherapy.

To allow the conduit to pass freely into the chest and prevent obstruction and ischemia of the gastric conduit, the esophageal hiatus is frequently enlarged during an esophagectomy, which increases the potential for a hernia, thus allowing abdominal contents to pass into the chest. ${ }^{6}$ Therefore, iatrogenic enlargement of esophageal foramina plays a major role in the incidence of PDH. When DH occurs early, the cause is mainly due to a lack of peritoneal adhesions. ${ }^{4}$ With longer

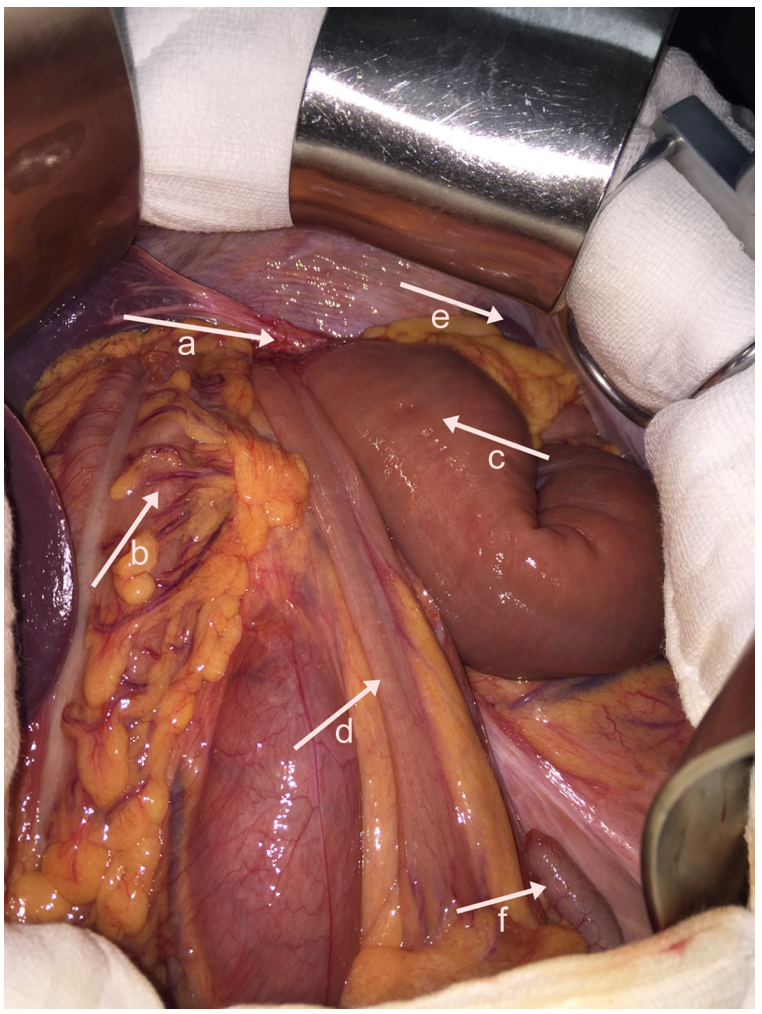

Figure 3 At exploratory laparotomy, the partial transverse colon with the total small bowel had herniated through the left esophageal hiatus into the left pleural cavity. a, esophageal hiatus; b, colon ascendens; c, proximal empty field; d, terminal ileum; e, spleen; and f, appendix.

follow-up during the postoperative period, different pressures between the thorax and the abdomen, increased intraabdominal pressure, and the negative pressure of the chest are predisposing factors that can contribute to abdominal contents passing into the chest. ${ }^{3}$ In addition, Ganeshan et al analyzed 440 postoperative patients with esophageal cancer and found that the type of approach to resection affects the incidence of PDH; transhiatal esophagectomy was associated with the highest incidence of PDH compared to other types of esophagectomy. ${ }^{7}$ In the current case, the esophagus was removed through the left thorax, the esophageal hiatus was enlarged, and gastric pull-through was performed with intrathoracic anastomosis. When the esophageal hiatus suture tore, the intestine easily herniated into the left pleura. Nausea and vomiting occurring with patients during chemotherapy can make the intra-abdominal pressure higher than the pressure in the chest. A high pressure in the abdominal cavity prompted the herniation of bowels into the chest. Herniation of the intestinal tract adjacent to the gastric conduit may result in bowel obstruction, pain, or colonic and small bowel ischemia.

The symptoms of PDH vary widely, depending on which organs herniate into the thoracic cavity, the degree of 
obstruction and twisting, and functional changes. Unfortunately, these patients always present with nonspecific clinical manifestations. ${ }^{8}$ Therefore, the diagnosis may be missed or delayed for some cases during the early period of a PDH. In the current case, the main patient complaint was nausea and vomiting; respiratory symptoms were not apparent, and no typical symptoms occurred. Recalling the early history and the response to chemotherapy in this patient, our preliminary consideration was related to the side effects caused by chemotherapy. Therefore, we did not focus enough attention on the early stage of the DH. This leads to some cases of misdiagnosis that causes serious complications, such as aspiration and intestinal necrosis.

Imaging examination is very important in the detection of this pathology. The diaphragmatic defect, hernia contents, and associated complications with other organ pathologies on images are important indices for the treatment of DH. Chest X-ray is used as the initial diagnostic tool for PDH in most institutions because of convenience, timeliness, and reproducibility, but chest X-ray has inevitable limitations for diagnostic accuracy. ${ }^{9}$ Barium enema may be used to diagnose thoracic herniation of the stomach, small intestine, and colon and is thus an important examination method. The shortcoming of a barium enema is retardation, which makes it difficult to establish an early diagnosis. Ultrasound (US) for useful tool for PDH because US shows diaphragmatic continuity and herniated organs; in addition, US reveals associated abdominal organ pathologies. An abdominal and thoracic CT scan is the most commonly used technique and reliable method in detecting a PDH and related complications because the time duration is short and a CT scan can be obtained easily and efficaciously in an emergency situation. The sensitivity and specificity of a CT scan for detecting DHs are $92 \%$ and $87 \%$, respectively. ${ }^{10}$ A multi-slice CT scan with coronal and sagittal reformatted images is the most useful and effective imaging technique for DHs. ${ }^{7}$ It can show the herniated abdominal organs together with complications, and the imaging time is shorter compared with a standard CT scan. Even though magnetic resonance (MR) imaging is not appropriate in most emergency cases, MR imaging can be used in chronic complicated cases due to the sensitivity for soft tissues. ${ }^{11}$ Taken together, imaging plays a crucial role in establishing the diagnosis of DH. For our presented case, the DH was diagnosed by preoperative CT and a barium meal. Laparotomy showed that the transverse colon (partial) and small intestine (total) had herniated through a wide esophageal hiatus into the chest cavity. A portion of the intestinal segments had signs of ischemia or gangrene. Thus, the DH described in this case report can be regarded as seldom seen and posing great danger to a patient.

PDH is rare but a potentially preventable event of esophagectomy. Ideally, the best approach would be to prevent DH in the first place after esophagectomy..$^{12}$ First, prevent respiratory infections to avoid severe cough after surgery. Second, if an intraoperative enlargement of the hiatus is necessary, an anterior incision can be performed in the diaphragm rather than a lateral incision, which is done to lower risk of herniation. ${ }^{13}$ Third, in particular, the hiatus should be inspected at the end of the operation; routine reduction of the hiatal defect with fixation of the gastric conduit at the hiatus is part of the optimization of the procedure for prevention of potential complications. ${ }^{14}$ Fourth, recovery restlessness and severe hiccups after general anesthesia should be reduced.

\section{Conclusion}

DH after esophagectomy is a relatively uncommon complication. In our case, incarcerated DH with bowel occlusion should be considered in the differential diagnosis in case of vomiting during adjuvant chemotherapy in patients who underwent esophagectomy for cancer. The associated side effects of chemotherapy similar to the early symptoms of $\mathrm{DH}$ can delay clinical diagnosis and are a greater risk to patients.

\section{Acknowledgment}

This work was supported by grants from Medical and Health Technology Development Program Foundation of Shandong Province, No 2015WS0149 and No 2015WS0197; Science and Technology Development Plan of Jinan, No 201401253; and Science and Technology Foundation of Shandong Academy of Medical Sciences, No 2013-45 and No 2014-04.

\section{Author contributions}

YS, JY, LY, and ZL were the treatment team. YS, JY, HX, and ZL performed data acquisition, analysis and interpretation, and critical revision of the manuscript. LY and HW were involved in data analysis and manuscript writing. All authors contributed toward data analysis, drafting and critically revising the paper and agree to be accountable for all aspects of the work.

\section{Disclosure}

The authors report no conflicts of interest in this work. 


\section{References}

1. Erkmen CP, Raman V, Ghushe ND, Trus TL. Laparoscopic repair of hiatal hernia after esophagectomy. J Gastrointest Surg. 2013;17(8): 1370-1374.

2. Daiko H, Nishimura M, Hayashi R. Diaphragmatic herniation after esophagectomy for carcinoma of the esophagus: a report of two cases. Esophagus. 2010;7:169-172.

3. Kent MS, Luketich JD, Tsai W, et al. Revisional surgery after esophagectomy: an analysis of 43 patients. Ann Thorac Surg. 2008;86(3):975-983.

4. Huang J, Zhou Y, Zhang H, et al. A phase II study of biweekly paclitaxel and cisplatin chemotherapy for recurrent or metastatic esophageal squamous cell carcinoma: ERCC1 expression predicts response to chemotherapy. Med Oncol. 2013;30(1):343.

5. Sun C, Shi HC, Wang K. Diaphragmatic hernia complicated with intestinal obstruction with colon perforation after surgery for esophageal cancer: a case report. Chin German J Clin Oncol. 2012;11:491-492.

6. van Sandick JW, Knegjens JL, van Lanschot JJ, Obertop H. Diaphragmatic herniation following oesophagectomy. Br J Surg. 1999;86(1): 109-112.

7. Ganeshan DM, Correa AM, Bhosale P, et al. Diaphragmatic hernia after esophagectomy in 440 patients with long-term follow-up. Ann Thorac Surg. 2013;96(4):1138-1145.
8. Choi YU, North JH Jr. Diaphragmatic hernia after Ivor-Lewis esophagectomy manifested as lower gastrointestinal bleeding. Am Surg. 2001; 67(1):30-32.

9. Eren S, Ciriş F. Diaphragmatic hernia: diagnostic approaches with review of the literature. Eur J Radiol. 2005;54(3):448-459.

10. Aly A, Watson DI. Diaphragmatic hernia after minimally invasive esophagectomy. Dis Esophagus. 2004;17(2):183-186.

11. Scaglione M, Pinto F, Grassi R, et al. Diagnostic sensitivity of computerized tomography in closed trauma of the diaphragm. Retrospective study of 35 consecutive cases. Radiol Med. 2000;99:46-50.

12. Cordero JA Jr, Moores DW. Thoracic herniation of the transverse colon after transhiatal esophagectomy. J Thorac Cardiovasc Surg. 2000; $120(2): 416$.

13. Vallböhmer D, Hölscher AH, Herbold T, Gutschow C, Schröder W. Diaphragmatic hernia after conventional or laparoscopic-assisted transthoracic esophagectomy. Ann Thorac Surg. 2007;84(6):1847-1852.

14. Hölscher AH, Vallböhmer D, Brabender J. The prevention and management of perioperative complications. Best Pract Res Clin Gastroenterol. 2006;20(5):907-923.
Therapeutics and Clinical Risk Management

\section{Publish your work in this journal}

Therapeutics and Clinical Risk Management is an international, peerreviewed journal of clinical therapeutics and risk management, focusing on concise rapid reporting of clinical studies in all therapeutic areas outcomes, safety, and programs for the effective, safe, and sustained use of medicines. This journal is indexed on PubMed Central, CAS,

\section{Dovepress}

EMBase, Scopus and the Elsevier Bibliographic databases. The manuscript management system is completely online and includes a very quick and fair peer-review system, which is all easy to use. Visit http://www.dovepress.com/testimonials.php to read real quotes from published authors.

Submit your manuscript here: http://www.dovepress.com/therapeutics-and-clinical-risk-management-journal 